\title{
Chain Stiffness and Excluded-Volume Effects in Polyelectrolyte Solutions: Characterization of Sodium Poly(2-acrylamido-2-methylpropanesulfonate) in Aqueous Sodium Chloride
}

\author{
Junko YASHIRO, Ryo HAgINO, Sachi SATO, and Takashi NORISUYE ${ }^{\dagger}$ \\ Department of Macromolecular Science, Graduate School of Science, Osaka University, \\ 1-1 Machikaneyama-cho, Toyonaka 560-0043, Japan
}

(Received July 28, 2005; Accepted August 31, 2005; Published January 15, 2006)

\begin{abstract}
Fourteen samples of sodium poly(2-acrylamido-2-methylpropanesulfonate) ranging in weight-average molecular weight from $1.7 \times 10^{3}$ to $1.4 \times 10^{6}$ have been studied by static light scattering (or sedimentation equilibrium) and viscometry to characterize the polyelectrolyte in 0.05 and $0.5 \mathrm{M}$ aqueous $\mathrm{NaCl}$ at $25^{\circ} \mathrm{C}$. The measured intrinsic viscosities (except for the two lowest molecular weights) and radii of gyration in the respective solvents are consistently described by combinations of the theories for unperturbed wormlike chains and excluded-volume effects (in the quasi-two-parameter scheme) with the parameter sets: $q$ (the total persistence length) $=1.5 \mathrm{~nm}, M_{\mathrm{L}}$ (the linear mass density) $=900 \mathrm{~nm}^{-1}, d$ (the chain diameter) $=1.6 \mathrm{~nm}$, and $B$ (the excluded-volume strength) $=3 \mathrm{~nm}$ in $0.5 \mathrm{M}$ aqueous $\mathrm{NaCl}$ and $q=3.0 \mathrm{~nm}, M_{\mathrm{L}}=900 \mathrm{~nm}^{-1}, d=1.7 \mathrm{~nm}$, and $B=6.2 \mathrm{~nm}$ in $0.05 \mathrm{M}$ aqueous $\mathrm{NaCl}$. It is shown that the end effect on the electrostatic persistence length hardly affects the estimation of $q$ in the aqueous salts. [DOI 10.1295/polymj.38.57]

KEY WORDS Polyelectrolyte / Poly(2-acrylamido-2-methylpropanesulfonate) / Wormlike Chain / Chain Stiffness / Electrostatic Persistence Length / Excluded-Volume Effect /
\end{abstract}

Although much experimental work has been done on the electrostatic persistence length ${ }^{1-4} q_{\mathrm{el}}$ for charged linear polymers in aqueous salts, our understanding of it still leaves much to be desired, at least, for intrinsically flexible or weakly stiff polyelectrolytes. ${ }^{5-8}$ The major problem in the estimation of $q_{\mathrm{el}}$ is that excluded-volume and stiffness effects can hardly be separated for those polymers without resort to a relevant excluded-volume theory. In previous studies, ${ }^{5-11}$ we utilized the quasi-two-parameter (QTP) theory (the Yamakawa-Stockmayer-Shimada theory) ${ }^{12-14}$ for the wormlike chain ${ }^{15}$ or, more generally, the helical wormlike chain ${ }^{14}$ to estimate these effects in aqueous $\mathrm{NaCl}$ solutions of sodium hyaluronate and sodium poly(styrenesulfonate) (Na PSS). Except for some details, the QTP scheme satisfactorily explained the molecular weight dependence of intrinsic viscosity $[\eta]$ and mean-square radius of gyration $\left\langle S^{2}\right\rangle$ for the two polyelectrolytes at fixed salt concentrations $C_{\mathrm{s}}$ higher than $10^{-2} \mathrm{M}$. On the other hand, the available polyelectrolyte theories ${ }^{1-4,16}$ failed to describe the $C_{\mathrm{s}}$ dependence of the estimated $q_{\mathrm{el}}$ and excluded-volume strength, though the degree of disagreement appeared to depend on the intrinsic chain stiffness. For deepening our understanding or drawing more general conclusions, we deemed it necessary to extend the work to other intrinsically flexible polyelectrolytes.

In the present work, with sodium poly(2-acryl- amido-2-methylpropanesulfonate) (Na PAMPS) chosen as such a polymer (see Figure 1), we made static light scattering (or sedimentation equilibrium) and viscosity measurements in aqueous $\mathrm{NaCl}$ with $C_{\mathrm{s}}$ of 0.05 and $0.5 \mathrm{M}$ at $25^{\circ} \mathrm{C}$ over a broad range of molecular weight from $1.7 \times 10^{3}$ to $1.4 \times 10^{6}$. The primary purpose here is to characterize the polyelectrolyte at these salt concentrations in terms of the wormlike chain model as a first step to the investigation of the ionic strength dependence of $q_{\mathrm{el}}$ and excluded-volume strength. With regard to this characterization work, the following remarks are in order.

Almost 30 years ago, Fisher et al. ${ }^{17,18}$ studied aqueous salt solutions of PAMPS by static light scattering and viscometry. Although their work was extensive in terms of the variety of aqueous solvents and PAMPS<smiles>CCCC(=O)NC(C)(C)CS(=O)(=O)O</smiles>

Figure 1. Repeating unit of sodium poly(2-acrylamido-2methylpropanesulfonate) (Na PAMPS).

${ }^{\dagger}$ To whom correspondence should be addressed (E-mail: norisuye@chem.sci.osaka-u.ac.jp). 
salts investigated, the samples were limited to a narrow molecular weight range from $1.4 \times 10^{5}$ to $8.7 \times$ $10^{5}$ (for example, for Na PAPMS). A point to note is that molecular characteristics of flexible chains can hardly be extracted from solution data in such a narrow and high molecular weight range because they are usually concealed by excluded-volume effects. ${ }^{14}$ In fact, Fisher et al. ${ }^{18}$ only estimated apparent values for the (total) persistence length on the unacceptable assumption that the PAMPS chain in any aqueous salt is unperturbed by excluded-volume effect. As far as we know, no other solution characterization has been reported for PAMPS after the work of Fisher et al.

\section{EXPERIMENTAL}

\section{Preparation of Samples}

Acid form PAMPS samples were prepared by the radical polymerization of 2-acrylamido-2-methylpropanesulfonic acid (AMPS) (about $10 \mathrm{wt} \%$ in concentration) in water, methanol, or ethanol at about $60^{\circ} \mathrm{C}$ with azobis(isobutyronitrile) or ammonium peroxodisulfate (5-10\% per monomer) as the initiator. The polymer was reprecipitated into $95 \%$ aqueous acetone (from water solutions with $0.1 \mathrm{M} \mathrm{NaCl}$ added), pure acetone (from methanol solutions), or heptane (from ethanol solutions); in some cases, mixtures were centrifuged to obtain precipitates. Each product was washed with 95\% aqueous acetone three times (for the precipitate from aqueous $\mathrm{NaCl}$ ), pure acetone three times, and dried in vacuum. The AMPS monomer used was recrystallized from ethanol solutions $\left(20-60^{\circ} \mathrm{C}\right)$.

Seventeen PAPMS samples (102 g) prepared in this way were stored in a refrigerator after being converted to the Na salt form and freeze-dried (see below). Their intrinsic viscosities in $0.5 \mathrm{M}$ aqueous $\mathrm{NaCl}$ at $25^{\circ} \mathrm{C}$ ranged from 3.9 to $144 \mathrm{~cm}^{3} \mathrm{~g}^{-1}$. Some of them with appropriate $[\eta]$ were each divided into several parts by fractional precipitation with $0.1 \mathrm{M}$ aqueous sodium acetate as the solvent and acetone as the precipitant. Fractions having nearly identical $[\eta]$ were combined and again fractionated. This procedure was further repeated 2-3 times, and 14 fractions (designated below as $f-1, f-2, \ldots, f-14$ in the order of decreasing molecular weight) were chosen for the present work.

Each of these fractions, dissolved in deionized water, was passed through a mixed-bed ion exchanger (Amberlite IR-120 + IR-400). The solution ( $\mathrm{pH} \sim 2$ ) was then neutralized with 0.05 or $0.1 \mathrm{~N}$ aqueous $\mathrm{NaOH}$, its $\mathrm{pH}$ being monitored by a Beckman $\phi 70 \mathrm{pH}$ meter. All the Na salt (Na PAMPS) samples obtained in this way were freeze-dried over more than $4 \mathrm{~d}$, and further dried in vacuum for a few days before use. Test solutions were prepared by dissolving a given Na PAMPS sample directly in 0.05 or $0.5 \mathrm{M}$ aqueous
$\mathrm{NaCl}$. The polymer mass concentration $c$ was calculated from the gravimetrically determined weight fraction with the solution density $\rho$.

\section{Light Scattering}

Scattering intensities were measured for 0.05 and $0.5 \mathrm{M}$ aqueous $\mathrm{NaCl}$ solutions of Na PAMPS samples f-1 through $\mathrm{f}-8$ at $25^{\circ} \mathrm{C}$ on a Fica 50 -light scattering photogoniometer with vertically polarized incident light of $436 \mathrm{~nm}$ in an angular range from 22.5 (or 30 ) to $150^{\circ}$. Pure benzene at $25^{\circ} \mathrm{C}$ was used to calibrate the apparatus with the Rayleigh ratio ${ }^{19}$ taken as $46.5 \times 10^{-6} \mathrm{~cm}^{-1}$ and its depolarization ratio was determined to be 0.420 by the method of Rubingh and $\mathrm{Yu}^{20}$ No correction for optical anisotropy was needed for the samples studied by light scattering. The intensity data obtained were analyzed by use of the plots of $\left(K c / R_{\theta}\right)^{1 / 2}$ vs. $c$ and $\left(K c / R_{\theta}\right)^{1 / 2} v s \cdot \sin ^{2}(\theta / 2)$ to evaluate the weight-average molecular weight $M_{\mathrm{w}}$, the second virial coefficient $A_{2}$, and $\left\langle S^{2}\right\rangle_{\mathrm{z}}$ (the z-average $\left\langle S^{2}\right\rangle$ ). Here, $K$ is the optical constant and $R_{\theta}$ is the excess reduced scattering intensity at scattering angle $\theta$. Test solutions were made optically clean by filtration through $0.45-\mu \mathrm{m}$ Millipore filters, followed by centrifugation at $2.5 \times 10^{4} \mathrm{~g}$ for $4 \mathrm{~h}$ in a Sorvall RC $5 \mathrm{C}$ centrifuge. The middle portion of each solution in the centrifuge tube was transferred directly into a light scattering cell with a carefully cleaned pipette. The cell and the pipette had been rinsed with refluxing acetone vapor for about $6 \mathrm{~h}$.

Excess refractive indices of aqueous $\mathrm{NaCl}$ solutions of Na PAMPS were measured at $25^{\circ} \mathrm{C}$ for the wavelengths $\lambda_{0}$ of 436 and $546 \mathrm{~nm}$ using a modified Schulz-Cantow type differential refractometer. Data for undialyzed $0.05 \mathrm{M} \mathrm{NaCl}$ solutions of samples with viscosity-average molecular weights $M_{\mathrm{v}}$ of $10^{5}$ and $3 \times 10^{3}$ showed the specific refractive index increments $(\partial n / \partial c)$ of Na PAMPS to be essentially independent of molecular weight in the range of our interest. This was also the case with the partial specific volume or the density increment $(\partial \rho / \partial c)$ in $0.5 \mathrm{M}$ aqueous $\mathrm{NaCl}$ (see below for the measurement of $\rho$ ). Based on these findings, 0.05 and $0.5 \mathrm{M}$ aqueous $\mathrm{NaCl}$ solutions of the sample with $M_{\mathrm{v}}=10^{5}$ were dialyzed against the corresponding solvents at $25^{\circ} \mathrm{C}$ using the previously described apparatus. ${ }^{21}$ The $(\partial n / \partial c)_{\mu}$ values obtained for the dialyzed solutions (i.e., at fixed chemical potentials $\mu$ of diffusible components) at $25^{\circ} \mathrm{C}$ were 0.146 and $0.143 \mathrm{~cm}^{3} \mathrm{~g}^{-1}$ at $C_{\mathrm{s}}=0.05 \mathrm{M}$ for 436 and $546 \mathrm{~nm}$, respectively, and 0.141 and $0.139 \mathrm{~cm}^{3} \mathrm{~g}^{-1}$ at $C_{\mathrm{s}}=0.5 \mathrm{M}$ for 436 and $546 \mathrm{~nm}$, respectively.

\section{Sedimentation Equilibrium}

Weight-average molecular weights and $\mathrm{z}$ - to weight-average molecular weight ratios $\left(M_{\mathrm{z}} / M_{\mathrm{w}}\right)$ for 
samples f-9-f-14 were determined by sedimentation equilibrium with $0.5 \mathrm{M}$ aqueous $\mathrm{NaCl}$ at $25^{\circ} \mathrm{C}$ as the solvent (see ref 22 for the procedure of data analysis). Use was made of a Beckman Optima XL-I ultracentrifuge with a diode laser of $\lambda_{0}=675 \mathrm{~nm}$ and an aluminum 12-mm double-sector cell. The solution column height was adjusted to about $2.5 \mathrm{~mm}$ and the rotor speed, to $11000-35000 \mathrm{rpm}$ depending on sample's molecular weight. The observed equilibrium sedimentation patterns for $\lambda_{0}=675 \mathrm{~nm}$ were converted to the concentration profiles using a $(\partial n / \partial c)_{\mu}$ value of 0.137 $\mathrm{cm}^{3} \mathrm{~g}^{-1}$, which was estimated by extrapolation of the aforementioned experimental values at 436 and 546 to $675 \mathrm{~nm}$ using a plot of $(\partial n / \partial c)_{\mu}$ against $\lambda_{0}{ }^{-2}$.

Densities of 0.05 and $0.5 \mathrm{M}$ aqueous $\mathrm{NaCl}$ solutions of Na PAMPS at $25^{\circ} \mathrm{C}$ were determined with an Anton Paar DMA5000 densitometer. The values of $(\partial \rho / \partial c)_{\mu}$ obtained for dialyzed solutions were 0.381 and 0.374 at $C_{\mathrm{s}}=0.05$ and $0.5 \mathrm{M}$, respectively.

\section{Viscometry}

Viscosities of 0.05 and $0.5 \mathrm{M}$ aqueous $\mathrm{NaCl}$ solutions of all Na PAMPS samples at $25^{\circ} \mathrm{C}$ were measured using conventional capillary viscometers of the Ubbelohde type; shear rate effects were negligible even for the highest molecular weight sample studied. The difference between the solution and solvent densities was taken into account in evaluation of the relative viscosity $\eta_{\mathrm{r}}$, using the measured $\partial \rho / \partial c$ values of 0.399 and 0.394 for undialyzed solutions of 0.05 and $0.5 \mathrm{M} \mathrm{NaCl}$, respectively.

The Huggins constant, normal down to $M_{\mathrm{w}} \sim 10^{5}$ in either aqueous salt, increased beyond 0.5 with lowering molecular weight and exceeded unity for $M_{\mathrm{w}}$ below $8 \times 10^{3}$. In particular, for the two lowest $M_{\mathrm{w}}$-samples the Huggins and Mead-Fuoss plots had no definite linear portion, making extrapolation of $\eta_{\mathrm{sp}} / c$ or $\left(\ln \eta_{\mathrm{r}}\right) / c$ to infinite dilution ambiguous (the specific viscosity $\eta_{\mathrm{sp}}$ was measured down to 0.060.07 ). The $[\eta]$ data for these two samples (given in the next section) may be less accurate. We previously encountered similar (or more serious) difficulty in determining $[\eta]$ for Na PSS samples of low molecular weight in aqueous $\mathrm{NaCl}$ at $C_{\mathrm{s}}=0.005$ and $0.01 \mathrm{M}$ (see Figure 1 of ref 7).

\section{RESULTS AND DISCUSSION}

\section{Molecular Weight, Radius of Gyration, and Intrinsic Viscosity}

Figure 2 illustrates the angular dependence of $(K c /$ $\left.R_{\theta}\right)_{c=0}^{1 / 2}$, i.e., $\left(K c / R_{\theta}\right)^{1 / 2}$ at infinite dilution, for $\mathrm{Na}$ PAMPS samples f-1-f-8 in $0.05 \mathrm{M}$ aqueous $\mathrm{NaCl}$ at $25^{\circ} \mathrm{C}$. The intercepts and (initial) slopes of the indicated straight lines give $M_{\mathrm{w}}$ and $\left\langle S^{2}\right\rangle_{\mathrm{z}}$ for the respec-

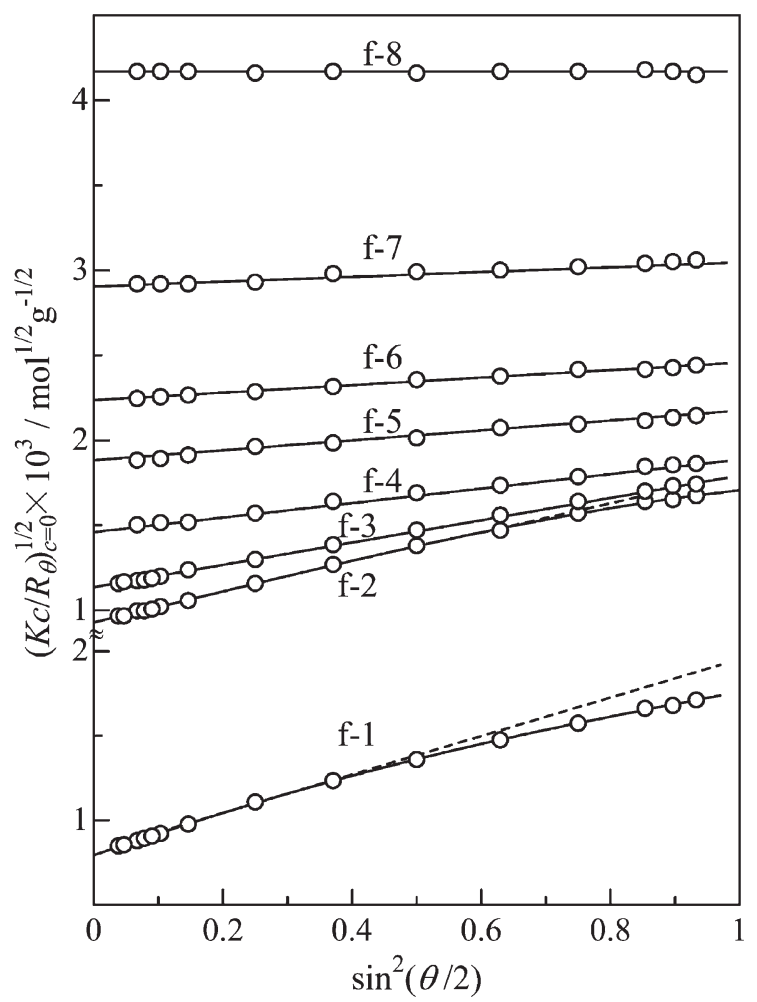

Figure 2. Angular dependence of $\left(K c / R_{\theta}\right)_{c=0}^{1 / 2}$ for $\mathrm{Na}$ PAMPS samples f- 1 through $\mathrm{f}-8$ in $0.05 \mathrm{M}$ aqueous $\mathrm{NaCl}$ at $25^{\circ} \mathrm{C}$.

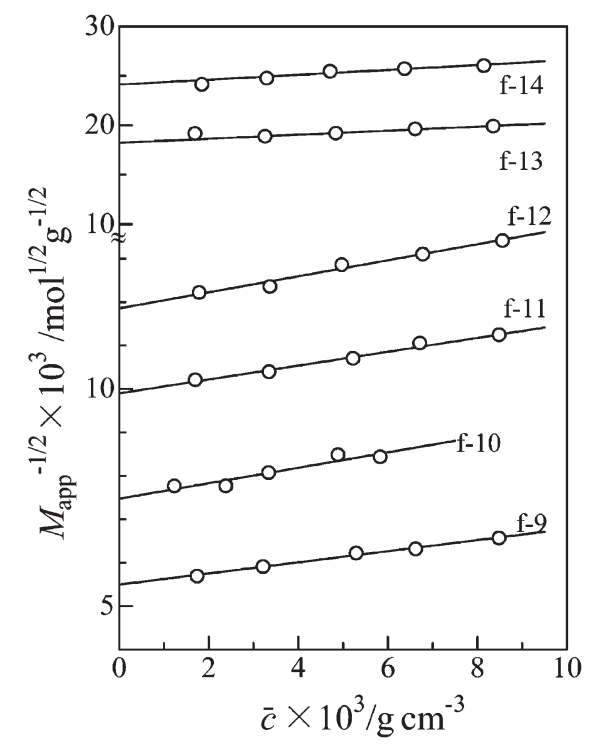

Figure 3. Plots of $M_{\text {app }}{ }^{-1 / 2}$ against $\bar{c}$ for Na PAMPS samples $\mathrm{f}-9-\mathrm{f}-14$ in $0.5 \mathrm{M}$ aqueous $\mathrm{NaCl}$ at $25^{\circ} \mathrm{C}$.

tive samples. Figure 3 shows the plots of $M_{\text {app }}^{-1 / 2}$ against $\bar{c}$ constructed from sedimentation equilibrium data for samples f-9-f-14 in $0.5 \mathrm{M}$ aqueous $\mathrm{NaCl}$ at $25^{\circ} \mathrm{C}$, where $M_{\text {app }}$ is the apparent molecular weight and $\bar{c}$ denotes the mean equilibrium concentrations at the liquid meniscus and the cell bottom. The numerical data for $M_{\mathrm{w}},\left\langle S^{2}\right\rangle_{\mathrm{z}}$, and $A_{2}$ obtained are summarized in Table I, along with those for $M_{\mathrm{z}} / M_{\mathrm{w}}$ and [ $\left.\eta\right]$. The molecular weight ratios indicate that our samples 
Table I. Results from light scattering, sedimentation equilibrium, and viscosity measurements on Na PAMPS samples in 0.5 and $0.05 \mathrm{M}$ aqueous $\mathrm{NaCl}$ at $25^{\circ} \mathrm{C}$

\begin{tabular}{|c|c|c|c|c|c|c|c|c|c|}
\hline \multirow[b]{2}{*}{ Sample } & \multicolumn{5}{|c|}{$0.5 \mathrm{M} \mathrm{NaCl}$} & \multicolumn{4}{|c|}{$0.05 \mathrm{M} \mathrm{NaCl}$} \\
\hline & $10^{-4} M_{\mathrm{w}}$ & $\begin{array}{c}10^{4} A_{2} \\
\left(\mathrm{~mol} \mathrm{~g}{ }^{-2} \mathrm{~cm}^{3}\right)\end{array}$ & $M_{\mathrm{z}} / M_{\mathrm{w}}$ & $\begin{array}{l}\left\langle S^{2}\right\rangle_{\mathrm{z}} \\
\left(\mathrm{nm}^{2}\right)\end{array}$ & $\begin{array}{c}{[\eta]} \\
\left(\mathrm{cm}^{3} \mathrm{~g}^{-1}\right)\end{array}$ & $10^{-4} M_{\mathrm{w}}$ & $\begin{array}{c}10^{4} A_{2} \\
\left(\mathrm{~mol} \mathrm{~g}{ }^{-2} \mathrm{~cm}^{3}\right)\end{array}$ & $\begin{array}{l}\left\langle S^{2}\right\rangle_{\mathrm{z}} \\
\left(\mathrm{nm}^{2}\right)\end{array}$ & $\begin{array}{c}{[\eta]} \\
\left(\mathrm{cm}^{3} \mathrm{~g}^{-1}\right)\end{array}$ \\
\hline$f-1$ & 144 & 2.25 & & 3090 & 261 & 150 & 4.85 & 5610 & 616 \\
\hline$f-2$ & 114 & 2.53 & & 2340 & 220 & 114 & 6.35 & 3910 & 495 \\
\hline$f-3$ & 76.0 & 2.83 & & 1400 & 154 & 77.5 & 7.38 & 2430 & 362 \\
\hline$f-4$ & 45.7 & 3.21 & & 770 & 106 & 46.9 & 8.31 & 1250 & 212 \\
\hline$f-5$ & 26.9 & 3.69 & & 380 & 70.7 & 28.2 & 10.3 & 660 & 143 \\
\hline$f-6$ & 19.2 & 4.08 & & 250 & 52.8 & 20.0 & 11.6 & 430 & 101 \\
\hline$f-7$ & 11.2 & 4.84 & & 130 & 34.6 & 11.8 & 14.3 & 230 & 64.5 \\
\hline $\mathrm{f}-8$ & 5.54 & 6.50 & & & 20.8 & 5.75 & 19.2 & & 35.8 \\
\hline$f-9$ & $3.31^{\mathrm{a}}$ & $7.04^{\mathrm{a}}$ & $1.1_{5}^{\mathrm{a}}$ & & 13.5 & & & & 19.7 \\
\hline$f-10$ & $1.79^{\mathrm{a}}$ & $13.3^{\mathrm{a}}$ & $1.1^{\mathrm{a}}$ & & 9.55 & & & & 13.7 \\
\hline$f-11$ & $1.02^{\mathrm{a}}$ & $15.8^{\mathrm{a}}$ & $1.1^{\mathrm{a}}$ & & 6.50 & & & & 8.65 \\
\hline$f-12$ & $0.711^{\mathrm{a}}$ & $21.9^{\mathrm{a}}$ & $1.1_{5}^{\mathrm{a}}$ & & 4.95 & & & & 6.36 \\
\hline$f-13$ & $0.301^{\mathrm{a}}$ & $37.4^{\mathrm{a}}$ & $1.2^{\mathrm{a}}$ & & 3.92 & & & & 5.03 \\
\hline$f-14$ & $0.172^{\mathrm{a}}$ & $59.7^{\mathrm{a}}$ & $1.0_{5}^{\mathrm{a}}$ & & 2.80 & & & & 3.99 \\
\hline
\end{tabular}

${ }^{a}$ From sedimentation equilibrium.

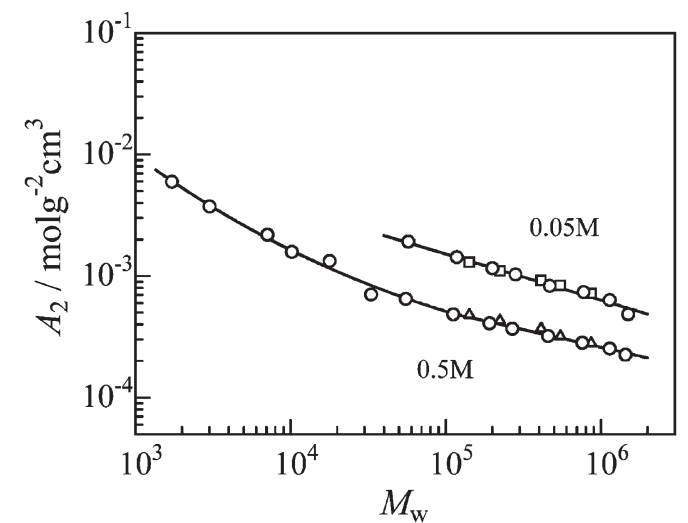

Figure 4. Molecular weight dependence of $A_{2}$ (circles) for $\mathrm{Na}$ PAMPS in 0.05 and $0.5 \mathrm{M}$ aqueous $\mathrm{NaCl}$ at $25^{\circ} \mathrm{C}$. The squares and triangles represent the data of Fisher et al. ${ }^{17}$

are fairly narrow in molecular weight distribution. The values of $M_{\mathrm{w}}$ from light scattering at $0.05 \mathrm{M}$ are systematically larger than those at $0.5 \mathrm{M}$, but the discrepancy is less than $\pm 2.7 \%$.

The molecular weight dependence of $A_{2}$ and that of $\left\langle S^{2}\right\rangle_{\mathrm{z}}$ in the aqueous salts are depicted in Figures 4 and 5 , respectively, both of which include the data of Fisher et al. ${ }^{17}$ In the former figure, the present and earlier data at either $C_{\mathrm{s}}$ form a curve that is essentially linear for $M_{\mathrm{w}}>5 \times 10^{4}$ with a slope of -0.30 for $C_{\mathrm{s}}=0.5 \mathrm{M}$ and -0.38 for $C_{\mathrm{s}}=0.05 \mathrm{M}$. On the other hand, the two data sets in the latter figure considerably deviate from each other. Ours are each fitted by a straight line with slope 1.23 for $C_{\mathrm{s}}=0.5 \mathrm{M}$ and 1.27 for $C_{\mathrm{s}}=0.05 \mathrm{M}$, whereas Fisher et al.'s $\left\langle S^{2}\right\rangle_{\mathrm{z}}$ values (except ones for $M_{\mathrm{w}}=1.4 \times 10^{5}$ ) are proportional to $M_{\mathrm{w}}$ at both $\mathrm{NaCl}$ concentrations as if the chains were Gaussian. This Gaussian-like behavior

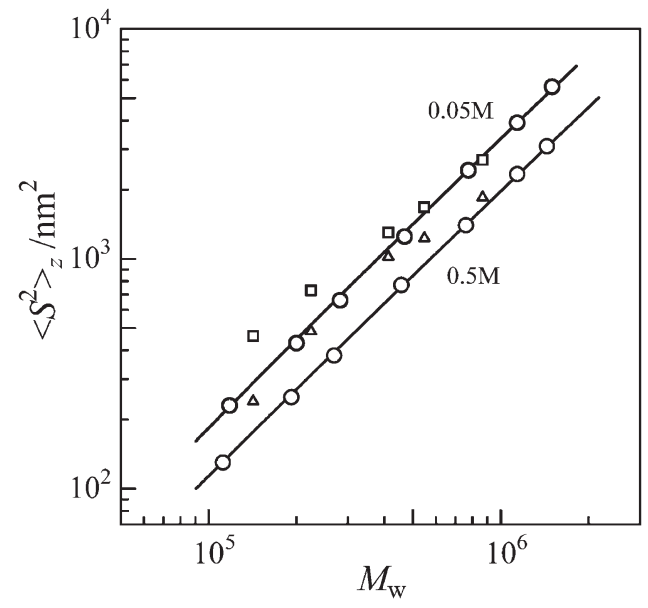

Figure 5. Molecular weight dependence of $\left\langle S^{2}\right\rangle_{z}$ (circles) for Na PAMPS in 0.05 and $0.5 \mathrm{M}$ aqueous $\mathrm{NaCl}$ at $25^{\circ} \mathrm{C}$, compared with the theoretical curves (eqs 7 and 8) for perturbed wormlike chains with the parameters in Table II and $M_{\mathrm{z}} / M_{\mathrm{w}}=1.1$. The squares and triangles represent the data of Fisher et al. ${ }^{17}$

of $\left\langle S^{2}\right\rangle_{z}$ seems inconsistent with the large $A_{2}$ values, which suggest that the chains should be perturbed by large excluded-volume effects. We note that our exponents 1.23 and 1.27 are comparable to or slightly larger than the value 1.2 for nonionic flexible chains in the good solvent limit.

Figure 6 shows the molecular weight dependence of $[\eta]$ in the two aqueous salts. The solid curves fitting the data points in the respective solvents are essentially linear for $M_{\mathrm{w}}$ above $3 \times 10^{4}$ with viscosity exponents 0.78 for $C_{\mathrm{s}}=0.5 \mathrm{M}$ and 0.89 for $C_{\mathrm{s}}=0.05 \mathrm{M}$ and bend up with decreasing $M_{\mathrm{w}}$ below $2 \times 10^{4}$. The latter exponent also exceeds the limiting slope 0.80 for nonionic flexible chains. The dashed lines 


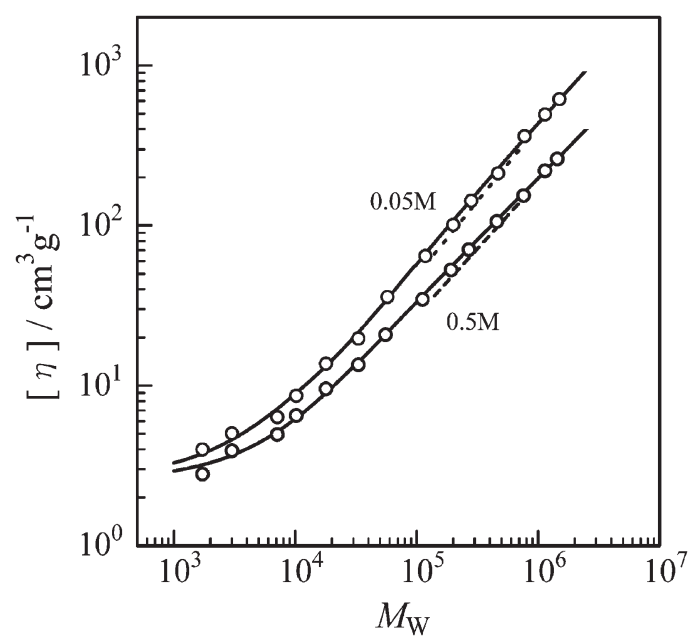

Figure 6. Molecular weight dependence of $[\eta]$ for Na PAMPS in 0.05 and $0.5 \mathrm{M}$ aqueous $\mathrm{NaCl}$ at $25^{\circ} \mathrm{C}$. The dashed lines represent the data of Fisher et al. ${ }^{17}$

representing the data of Fisher et al. ${ }^{17}$ come fairly close to our data points in the corresponding $M_{\mathrm{w}}$ range. To sum up, we can say that the Na PAMPS chain assumes a highly expanded or extended coil conformation at either $C_{\mathrm{s}}$.

\section{Data Analysis and Comparison with Theory}

We analyze the viscosity data in Figure 6 by a combination of Yoshizaki et al.'s theory ${ }^{23}$ for the intrinsic viscosity $[\eta]_{0}$ of an unperturbed wormlike touchedbead chain and the QTP theory ${ }^{12-14}$ for the viscosity expansion factor $\alpha_{\eta}$. The former theory contains three parameters, the (total) persistence length $q$, the contour length $L$, and the bead diameter $d$; $L$ is related to the molecular weight $M$ by $M / M_{\mathrm{L}}$, with $M_{\mathrm{L}}$ being the molar mass per unit contour length of the chain. In the QTP scheme, $\alpha_{\eta}{ }^{3}$ is expressed by

$$
\alpha_{\eta}{ }^{3}=\left(1+3.8 \tilde{z}+1.9 \tilde{z}^{2}\right)^{0.3}
$$

if the Barrett equation ${ }^{24}$ is adopted. Here, $\tilde{z}$ is the scaled excluded-volume parameter defined by ${ }^{13,14}$

$$
\tilde{z}=(3 / 4) K\left(L_{r}\right) z
$$

with

$$
\begin{aligned}
K\left(L_{r}\right)= & (4 / 3)-2.711\left(L_{r}\right)^{-1 / 2}+(7 / 6)\left(L_{r}\right)^{-1} \\
& \text { for } L_{r}>6 \\
= & \left(L_{r}\right)^{-1 / 2} \exp \left[-6.611\left(L_{r}\right)^{-1}\right. \\
+ & \left.0.9198+0.03516 L_{r}\right] \quad \text { for } L_{r} \leq 6 \\
& z=(3 / 2 \pi)^{3 / 2}(B / 2 q)\left(L_{r}\right)^{1 / 2}
\end{aligned}
$$

and

$$
L_{r}=L / 2 q
$$

The excluded-volume strength $B$ in eq 4 is defined (for the wormlike chain) by
Table II. Model parameters for Na PAMPS in 0.5 and $0.05 \mathrm{M}$ aqueous $\mathrm{NaCl}$ at $25^{\circ} \mathrm{C}$

\begin{tabular}{cccccc}
\hline$C_{\mathrm{s}} / \mathrm{M}$ & $M_{\mathrm{L}} / \mathrm{nm}^{-1}$ & $q / \mathrm{nm}$ & $d / \mathrm{nm}$ & $B / \mathrm{nm}$ & method \\
\hline 0.5 & $900^{\mathrm{a}}$ & 1.4 & 1.55 & 2.7 & {$[\eta]$} \\
& $900^{\mathrm{a}}$ & 1.6 & - & 3.0 & $\left\langle S^{2}\right\rangle$ \\
0.05 & $900^{\mathrm{a}}$ & 3.0 & 1.7 & 6.2 & {$[\eta]$} \\
& $900^{\mathrm{a}}$ & 3.0 & - & 6.2 & $\left\langle S^{2}\right\rangle$ \\
\hline
\end{tabular}

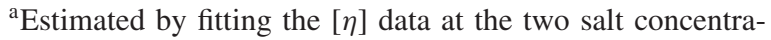
tions (see the text).

$$
B=\beta / a^{2}
$$

where $\beta$ and $a$ are the binary cluster integral and the bead spacing, respectively. It should be noted that in the coil limit, $\tilde{z}$ reduces to the conventional excluded-volume parameter $z$ in the two-parameter theory.

As may be seen from the above equations, $[\eta]$ $\left(=[\eta]_{0} \alpha_{\eta}{ }^{3}\right)$ for a given $M_{\mathrm{w}}$ in a nonideal solvent is determined by $M_{\mathrm{L}}, q, d$, and $B$, but all the four parameters for Na PAMPS in each aqueous $\mathrm{NaCl}$ cannot uniquely be determined from our $[\eta]$ data. In the present analysis, we fixed $M_{\mathrm{L}}$ in the two aqueous salts to $900 \mathrm{~nm}^{-1}$ and searched for a set of $q, d$, and $B$ leading to the best fit to the data for $M_{\mathrm{w}}>7 \times 10^{3}$ at the respective $C_{\mathrm{s}}$. The uncertainties of the three parameters thus obtained were of the order of $\pm 10 \%$. The $M_{\mathrm{L}}$ of $900 \mathrm{~nm}^{-1}$, close to the value $920 \mathrm{~nm}^{-1}$ expected for the polyelectrolyte chain in the all trans conformation, is based on the finding that, when simultaneous curve fitting was made to the data sets for $C_{\mathrm{s}}=0.05$ and $0.5 \mathrm{M}$ with $M_{\mathrm{L}}$ fixed to different arbitrary values, best (but indistinguishable) fits (for $M_{\mathrm{w}}>7 \times 10^{3}$ ) were obtained for $M_{\mathrm{L}}=900 \pm 100 \mathrm{~nm}^{-1}$.

The estimated parameters are summarized in Table II, and the theoretical curves for the two aqueous salts are compared with the data in Figure 7 (see the caption for the dashed-line portions). Except for the two lowest $-M_{\mathrm{w}}$ samples, the agreement is generally good. The observed deviations for these samples are most likely due to experimental errors arising from ambiguous extrapolation of $\eta_{\mathrm{sp}} / c$ or $\left(\ln \eta_{\mathrm{r}}\right) / c$ to $c=0$ (see the experimental section).

The solid lines closely fitting the circles in Figure 5 represent the theoretical $\left\langle S^{2}\right\rangle_{\mathrm{z}}$ values $\left(=\alpha_{\mathrm{s}}{ }^{2}\left\langle S^{2}\right\rangle_{0} M_{\mathrm{z}} /\right.$ $\left.M_{\mathrm{w}}\right)$ calculated from the expressions

$$
\begin{aligned}
\left\langle S^{2}\right\rangle_{0}= & (q L / 3)-q^{2}+\left(2 q^{3} / L\right) \\
& -\left(2 q^{4} / L^{2}\right)[1-\exp (-L / q)]
\end{aligned}
$$

for the unperturbed mean-square radius of gyration ${ }^{25}$ and

$$
\begin{aligned}
\alpha_{\mathrm{s}}^{2}= & {\left[1+10 \tilde{z}+\left(\frac{70 \pi}{9}+\frac{10}{3}\right) \tilde{z}^{2}+8 \pi^{3 / 2} \tilde{z}^{3}\right]^{2 / 15} } \\
& \times\left[0.933+0.067 \exp \left(-0.85 \tilde{z}-1.39 \tilde{z}^{2}\right)\right]
\end{aligned}
$$




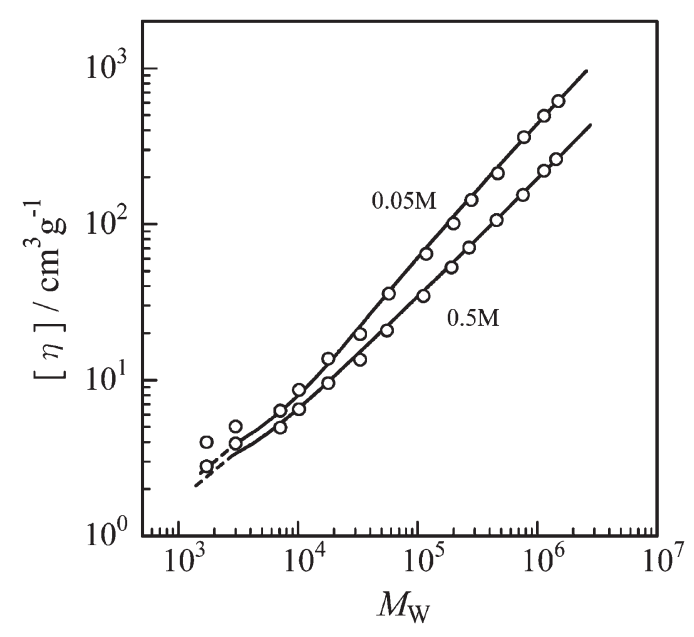

Figure 7. Comparison between the measured $[\eta]$ for $\mathrm{Na}$ PAMPS in 0.05 and $0.5 \mathrm{M}$ aqueous $\mathrm{NaCl}$ at $25^{\circ} \mathrm{C}$ and the theoretical curves computed from the theory of Yoshizaki et al. ${ }^{23}$ for unperturbed wormlike touched-bead chains and the QTP theory ${ }^{12-14}$ (eqs 1-6) for excluded-volume effects. For clarity, the theoretical values (discrete for low molecular weights) at the respective salt concentrations are represented by continuous solid lines and those for one and two beads are connected by dashed straight lines.

for the squared radius expansion factor $\alpha_{\mathrm{s}}{ }^{2}$ (the Domb-Barrett equation $^{26}$ in the QTP scheme) with $M_{\mathrm{z}} / M_{\mathrm{w}}=1.1$ (the mean of the values in Table I) and the parameters presented in Table II. The $q$ and $B$ values used have been altered slightly from those estimated above from $[\eta]$ (when necessary) so that eqs 7 and 8 with $M_{\mathrm{z}} / M_{\mathrm{w}}=1.1$ give the closest agreement with the data.

The parameters from $\left\langle S^{2}\right\rangle$ in Table II essentially agree with those from $[\eta]$, leading to the conclusion that the present $[\eta]$ and $\left\langle S^{2}\right\rangle_{\mathrm{z}}$ data for Na PAMPS in 0.05 and $0.5 \mathrm{M}$ aqueous $\mathrm{NaCl}$ are consistently explained in the framework of the QTP theory. If the parameters from $[\eta]$ are used for $\left\langle S^{2}\right\rangle_{z}$, the theoretical line for $C_{\mathrm{s}}=0.5 \mathrm{M}$ in Figure 5 shifts downward by about $8 \%$. The resulting discrepancy is plausible, in that Yoshizaki et al.'s theory ${ }^{23}$ in the coil limit, equivalent to the Kirkwood-Riseman theory, ${ }^{27}$ overestimates $[\eta]$ (or the Flory viscosity factor) by $3-22 \%{ }^{28,29}$ The perfect agreement between the parameter sets from $[\eta]$ and $\left\langle S^{2}\right\rangle_{\mathrm{z}}$ at $C_{\mathrm{s}}=0.05 \mathrm{M}$ may be somewhat fortuitous, even though the theoretical error diminishes as the chain becomes stiff. ${ }^{30}$ The unperturbed dimensions $\left\langle S^{2}\right\rangle_{0} / M\left(=q / 3 M_{\mathrm{L}}\right)$ at infinite $M$ are obtained as $0.60 \times 10^{-3}$ and $1.1 \times 10^{-3} \mathrm{~nm}^{2}$ in 0.5 and $0.05 \mathrm{M}$ aqueous $\mathrm{NaCl}$, respectively, both of which are roughly one-third the earlier estimates by Fisher et al. ${ }^{18}$

\section{Chain Stiffness and Excluded-Volume Effects}

The $q$ values for Na PAMPS in Table II are comparable to those reported previously ${ }^{8,11}$ for Na PSS in aqueous $\mathrm{NaCl}$ with the corresponding salt concentrations $\left(1.5 \mathrm{~nm}\right.$ at $C_{\mathrm{s}}=0.5 \mathrm{M}$ and $2.7 \mathrm{~nm}$ at $C_{\mathrm{s}}=0.05$ $\mathrm{M}$, both from $[\eta]$ ), indicating that the two polyelectrolytes have similar stiffness in the aqueous salts. On the other hand, the $B$ values for Na PAMPS are considerably larger than those for Na PSS $\left(1.6 \mathrm{~nm}\right.$ at $C_{\mathrm{s}}=0.5$ $\mathrm{M}$ and $4.0 \mathrm{~nm}$ at $C_{\mathrm{s}}=0.05 \mathrm{M}$ ). The difference may be due partly to stronger attractive interactions between phenylene groups in the latter polymer, but since it is more pronounced at the lower $C_{\mathrm{s}}$ of $0.05 \mathrm{M}$, the electrostatic contributions to $B$ must differ between the two polymers having essentially the same charge densities.

The Na PAMPS chain is appreciably stiffer at $C_{\mathrm{s}}=$ $0.05 \mathrm{M}$ than at $C_{\mathrm{s}}=0.5 \mathrm{M}$ in line with theoretical predictions ${ }^{1-4}$ for the electrostatic stiffening effect. As remarked by Hagerman ${ }^{31}$ on the basis of the OdijkSkolnick-Fixman (OSF) theory, ${ }^{1,2}$ however, the value of $q_{\mathrm{el}}$ and hence that of $q$ (the sum of $q_{\mathrm{el}}$ and the intrinsic persistence length $q_{0}$ ) for short chains can only be apparent owing to the diminished total electrostatic interaction energy on the polyelectrolyte chain. In other words, $q_{\mathrm{el} \text {,app }}$ (the apparent value of $q_{\mathrm{el}}$ at finite $L$ ) and $q_{\text {app }}$ (the apparent value of $q$, i.e., $q_{\text {app }}=q_{0}+$ $\left.q_{\mathrm{el}, \mathrm{app}}\right)$ equal to $q_{\mathrm{el}}$ and $q$, respectively, when $\kappa L \gg 1$, and both are decreasing functions of $\kappa L$ ( $\kappa$ denotes the reciprocal Debye length).

We examined this end effect on the electrostatic contribution to $q$ in the OSF scheme using

$$
q_{\text {app }}=q_{0}+\left(q-q_{0}\right) P
$$

where ${ }^{1,31}$

$$
\begin{aligned}
P \equiv & q_{\mathrm{el}, \mathrm{app}} / q_{\mathrm{el}}=1-(8 / 3)(\kappa L)^{-1} \\
& +(3 \kappa L)^{-1}\left(8+5 \kappa L+\kappa^{2} L^{2}\right) \exp (-\kappa L)
\end{aligned}
$$

The value of $q_{0}$ for Na PAMPS was roughly estimated to be $0.66 \mathrm{~nm}$ by linear extrapolation of $q$ (from $[\eta]) v s$. $C_{\mathrm{s}}{ }^{-1 / 2}$ to infinite ionic strength (an accurate $q_{0}$ is unnecessary for the present purpose). Equations 9 and 10 with this $q_{0}$ together with $q=3.0 \mathrm{~nm}$ and $M_{\mathrm{L}}=$ $900 \mathrm{~nm}^{-1}$ at $C_{\mathrm{s}}=0.05 \mathrm{M}$ (see Table II) yield $q_{\text {app }}=$ 2.85 and $1.62 \mathrm{~nm}$ for $M=5 \times 10^{4}$ and $5 \times 10^{3}$, respectively. Thus $q_{\text {app }}$ begins to deviate significantly from $q$ at $M=5 \times 10^{4}$ and becomes about one-half at $M=5 \times 10^{3}$. Except for very short chains, such decreases in (apparent) stiffness appreciably lower theoretical $[\eta]_{0}$, but they lead to increases in excluded-volume effect, thereby bringing theoretical $[\eta]$ $\left(=[\eta]_{0} \alpha_{\eta}{ }^{3}\right)$ back nearly to the original values given by $q$. The differences were at most a few \%; note that $[\eta]_{0}$ is insensitive to $q$ (or $q_{\text {app }}$ ) for very short chains, for which excluded-volume effects are negligible. At $0.5 \mathrm{M}, q_{\text {app }}$ appreciably decreases only for low $M$ and the effect on $[\eta]$ is insignificant throughout the entire 
molecular weight range of our interest. Thus, we may conclude that the estimation of $q$ for Na PAMPS in the aqueous salts is hardly affected by the diminution of the electrostatic interaction energy on the chain accompanying a decrease in $L$. This is similar to the earlier conclusion drawn for $\mathrm{Na}$ PSS. ${ }^{7}$

\section{CONCLUSIONS}

The molecular weight dependence of $[\eta]$ and that of $\left\langle S^{2}\right\rangle_{z}$ for Na PAMPS in 0.05 and $0.5 \mathrm{M}$ aqueous $\mathrm{NaCl}$ are described by the known expressions ${ }^{23-26}$ for unperturbed wormlike chains and excluded-volume effects in the quasi-two-parameter scheme ${ }^{12-14}$ with a degree of accuracy similar to that known for nonionic flexible polymers. ${ }^{14}$ The unperturbed dimensions $\left\langle S^{2}\right\rangle_{0} / M$ at infinite $M$ are $0.60 \times 10^{-3}$ and $1.1 \times 10^{-3} \mathrm{~nm}^{2}$ at $C_{\mathrm{s}}=0.5$ and $0.05 \mathrm{M}$, respectively. The (total) persistence lengths of $1.5( \pm 0.1) \mathrm{nm}$ at the higher $C_{\mathrm{s}}$ and $3.0 \mathrm{~nm}$ at the lower $C_{\mathrm{s}}$ are comparable to those for $\mathrm{Na} \mathrm{PSS}^{8,11}$ in the corresponding solvents, indicating that the two polyelectrolytes have similar chain stiffness. The electrostatic contributions to $q$ may also be similar, but discussions on $q_{\mathrm{el}}$ are deferred to the forthcoming paper, in which both $q_{\mathrm{el}}$ and the electrostatic excluded-volume strength for Na PAMPS in aqueous $\mathrm{NaCl}$ will be examined as functions of ionic strength.

\section{REFERENCES}

1. T. Odijk, J. Polym. Sci., Polym. Phys. Ed., 15, 477 (1977).

2. J. Skolnick and M. Fixman, Macromolecules, 10, 944 (1977).

3. M. Le Bret, J. Chem. Phys., 76, 6243 (1982).

4. M. Fixman, J. Chem. Phys., 76, 6346 (1982).

5. K. Hayashi, K. Tsutsumi, T. Norisuye, and A. Teramoto, Polym. J., 28, 922 (1996).

6. K. Tsutsumi and T. Norisuye, Polym. J., 30, 345 (1998).
7. Y. Iwamoto, E. Hirose, and T. Norisuye, Polym. J., 32, 428 (2000).

8. J. Yashiro and T. Norisuye, J. Polym. Sci., Part B: Polym. Phys., 40, 2728 (2002).

9. K. Hayashi, K. Tsutsumi, F. Nakajima, T. Norisuye, and A. Teramoto, Macromolecules, 28, 3824 (1995).

10. N. Mizukoshi and T. Norisuye, Polym. Bull., 40, 555 (1998).

11. E. Hirose, Y. Iwamoto, and T. Norisuye, Macromolecules, 32, 8629 (1999).

12. H. Yamakawa and W. H. Stockmayer, J. Chem. Phys., 57, 2843 (1972).

13. J. Shimada and H. Yamakawa, J. Chem. Phys., 85, 591 (1986).

14. H. Yamakawa, "Helical Wormlike Chains in Polymer Solutions," Springer, Berlin, 1997.

15. O. Kratky and G. Porod, Recl. Trav. Chim. Pays-Bas, 68, 1106 (1949).

16. M. Fixman and J. Skolnick, Macromolecules, 11, 863 (1978).

17. L. W. Fisher, A. R. Sochor, and J. S. Tan, Macromolecules, 10, 949 (1977).

18. L. W. Fisher, A. R. Sochor, and J. S. Tan, Macromolecules, 10, 955 (1977).

19. Gj. Dezelić and J. Vavra, Croat. Chem. Acta, 38, 35 (1966).

20. D. N. Rubingh and H. Yu, Macromolecules, 9, 681 (1976).

21. T. Sato, T. Norisuye, and H. Fujita, Macromolecules, 16, 185 (1983).

22. T. Norisuye, T. Yanaki, and H. Fujita, J. Polym. Sci., Polym. Phys. Ed., 18, 547 (1980).

23. T. Yoshizaki, I. Nitta, and H. Yamakawa, Macromolecules, 21, 165 (1988).

24. A. J. Barrett, Macromolecules, 17, 1566 (1984).

25. H. Benoit and P. Doty, J. Phys. Chem., 57, 958 (1953).

26. C. Domb and A. J. Barrett, Polymer, 17, 179 (1976).

27. J. G. Kirkwood and J. Riseman, J. Chem. Phys., 16, 565 (1948).

28. Y. Miyaki, Y. Einaga, H. Fujita, and M. Fukuda, Macromolecules, 13, 588 (1980).

29. T. Konishi, T. Yoshizaki, and H. Yamakawa, Macromolecules, 24, 5614 (1991).

30. T. Norisuye, Prog. Polym. Sci., 18, 519 (1993).

31. P. J. Hagerman, Biopolymers, 22, 811 (1983). 